\title{
Chronik der Medienentwicklung in Deutschland 2001
}

\author{
Hermann-Dieter Schröder
}

1. Medienregulierung (Medienpolitik / Medienrecht)

2. Medienunternehmen

3. Medienangebote

3.1 Presse

3.2 Rundfunk

3.3 Online-Medien

4. Werbung

5. Mediennutzung

\section{Medienregulierung (Medienpolitik / Medienrecht)}

Am 1. Januar tritt der novellierte Rundfunkstaatsvertrag ${ }^{1}$ in Kraft. Damit werden die Grundgebühr auf 5,32 Euro und die Fernsehgebühr auf 11,83 Euro monatlich erhöht. Computer mit Online-Anschluss sind bis Ende 2004 von der Rundfunkgebührenpflicht ausgenommen. Werbung ist auf den Teletext-Seiten der öffentlich-rechtlichen Rundfunkanstalten nicht mehr zugelassen. Auch das Gegendarstellungsrecht für das erste Fernsehprogramm ist neu geregelt: Es ist bei der Rundfunkanstalt durchzusetzen, die den betreffenden Beitrag zu verantworten hat.

Ebenfalls am 24. Januar verkündet das Bundesverfassungsgericht sein Urteil über die Verfassungsbeschwerden von n-tv gegen das seit 1964 bestehende Verbot von Fernsebaufnabmen in Gerichtsverhandlungen. Mit einer Mehrheit von 5:3 Stimmen wird die Beschwerde als nicht begründet zurückgewiesen. ${ }^{2}$

Am 24. Januar entscheidet das Verwaltungsgericht Hannover, dass der Beamtenbund in der Versammlung der Niedersächsischen Landesmedienanstalt (NLM) nicht vertreten sein kann, wenn er nicht gemäß Landesmediengesetz für mindestens jede zweite Amtszeit eine Frau entsendet. Der Beamtenbund hatte geltend gemacht, dass er keine Frau habe entsenden können, weil keine der dafür vorgeschlagenen Frauen aus dem Vorstand oder den Fachverbänden bereit gewesen sei, das Mandat zu übernehmen. Das Gericht hält dem entgegen, dass es nicht darum ginge, Verbandsinteressen zu vertreten, sondern die gesellschaftliche Vielfalt zu repräsentieren. Deshalb sei die Suche nach einer Vertreterin nicht auf die Leitungsebene des Verbandes zu beschränken.

Am 1. Februar wird der Streit zwischen der RTL Group und dem Leipziger Kabelnetzbetreiber Primacom beigelegt. Primacom hatte im Vorjahr begonnen, Programme wie ProSieben, RTL II und Vox nur noch gegen zusätzliches Entgelt in Digitalpaketen an Leipziger Haushalte zu verbreiten. Das Leipziger Landgericht hatte dieses Modell aus urheber- und vertragsrechtlichen Gründen bereits im Vorjahr untersagt. Gegenüber der RTL Group sichert Primacom nun zu, zunächst bis zum 31. März 2002 die Fernsehprogramme RTL, RTL II, Super RTL und Vox analog und im Basispaket primaTV auch digital zu verbreiten. Auch die Dienste von RTL New Media sollen digital verbreitet werden.

1 Abgedruckt in Funkkorrespondenz 1/2001.

2 Abgedruckt in epd medien 7/2002. 
Am 26. Februar weist der Verband Privater Rundfunk und Telekommunikation (VPRT) die Forderung der Gemeinsamen Stelle Jugendschutz und Programm der Landesmedienanstalten zurück, für so genannte Reality Soaps (auch Psychoformate genannt) freiwillige Verhaltensgrundsätze aufzustellen. Auch die von Politikern geforderte Einrichtung einer Kommission zur Ethik der Programmveranstalter sei nur dazu angetan, Verantwortlichkeiten $\mathrm{zu}$ verwischen. Unterdessen nimmt das Interesse der Zuschauer an solchen Sendungen deutlich ab; die meisten werden im Laufe des Jahres aus wirtschaftlichen Gründen eingestellt.

Am 1. März entscheidet der Bundesgerichtshof nach einem zehn Jahre andauernden Rechtsstreit, dass die ARD wegen ihrer Sendung „Tagesschau“ nicht verlangen kann, dass ähnliche Sendungstitel wie „Tagesreport“ (Sat.1) oder „Tagesbild“ (ProSieben) unterlassen werden.

Am 6. März entscheidet der Bundesgerichtshof, dass Kabelnetzbetreiber bei Preiserhöhungen nicht auf die Zustimmung von Wohnungsunternehmen angewiesen sind. Eine entsprechende Vertragsklausel ist nichtig.

Am 23. März weist der baden-württembergische Verwaltungsgerichtshof einen Eilantrag ab, mit dem die NPD durchsetzen wollte, dass der SWR einen von ihr produzierten fremdenfeindlichen Wablwerbespot senden muss.

Am 19. Juni entscheidet der 1. Senat des Bundesarbeitsgerichts, dass Redaktionsstatute als Bestandteil von Arbeitsverträgen zulässig sind. ${ }^{3}$ Beim Mannheimer Morgen war 1969 ein Redaktionsstatut eingeführt worden, das in der seit 1975 geltenden Fassung dem Redaktionsrat ein Mitbestimmungsrecht bei der Besetzung der Chefredaktion gab. 1996 hatte der Verlag das Statut gekündigt mit der Begründung, die Vereinbarung führe zu einer Einschränkung der verlegerischen Entscheidungs- und Pressefreiheit und verstoße gegen den im Betriebsverfassungsgesetz festgelegten Tendenzschutz. Das Gericht kommt zu dem Urteil, dass das Redaktionsstatut fortbesteht und nur mit Mitteln des Arbeitsvertragsrechts beendet werden kann.

Am 22. Juli beschließt die Sächsische Landesanstalt für privaten Rundfunk und neue Medien (SLM) eine Beanstandung auf der Grundlage der EU-Fernsehrichtlinie wegen pornografischer Inhalte des englischen Adult Channel. Das Programm wird in Leipzig vom Kabelnetzbetreiber Primacom mit einer digitalen Vorsperre verbreitet. Die Gemeinsame Stelle Jugendschutz und Programm der Arbeitsgemeinschaft der Landesmedienanstalten hat den Pornografieverdacht der SLM bestätigt. Da das Programm in England von der ITC zugelassen ist, wird zunächst bei der EU-Kommission angefragt, ob die von der SLM geplanten Maßnahmen mit dem Gemeinschaftsrecht vereinbar sind.

Am 19. September unterzeichnen die Repräsentanten von ARD, ZDF, RTL, der Kirch-Gruppe und der Direktorenkonferenz der Landesmedienanstalten (DLM) eine gemeinsame Erklärung der deutschen Programmveranstalter und der Landesmedienanstalten zur zügigen Einführung der Multimedia Home Platform (MHP) ${ }^{4}$ und verpflichten sich zur Einführung der MHP als Standard für interaktives Fernsehen. Danach sollen alle neuen interaktiven Mehrwertfunktionen auf dem MHP-Standard entwickelt werden, bis zum 1.7.2002 erste Dienste im MHP-Standard angeboten werden und alle bestehenden Dienste in absehbarer Zeit auf MHP überführt werden. Auf diese Weise soll auch für die neuen Kabelnetzbetreiber eine verbindliche Vorgabe erreicht werden.

3 Aktenzeichen 1 AZR 463/00.

4 Abgedruckt in epd medien 76/2001. 
Am 5. November veröffentlicht die EU-Kommission eine Mitteilung über die Anwendung der Vorschriften über staatliche Beibilfen auf den öffentlich-rechtlichen Rundfunk..$^{5}$ Die Kommission hält daran fest, dass sie für die Kontrolle staatlicher Beihilfen auch im Rundfunkbereich zuständig ist. Ein im Juni vorgelegter Entwurf hatte gefordert, der öffentliche Versorgungsauftrag solle gesetzlich eindeutig definiert werden. ARD, ZDF und der Bundesrat hatten eingewandt, die EU sei nicht befugt, Auftrag und Finanzierung des öffentlich-rechtlichen Rundfunks gemeinschaftsrechtlich zu definieren. Nunmehr ist nach Einschätzung deutscher Medienpolitiker hinreichend klargestellt, dass das in Deutschland praktizierte Verfahren der Gebührenfinanzierung nicht gegen die Regeln der EU verstößt.

Mit Urteil vom 6. Dezember untersagt der Bundesgerichtshof erneut eine Anzeige der Textilfirma Benetton. Sie genießt nicht den Schutz der Meinungsäußerungsfreiheit, weil sie die Menschenwürde Aids-Kranker verletzt und damit gegen Artikel 1 des Grundgesetzes verstößt. Eine frühere Entscheidung in dieser Sache war im Vorjahr vom Bundesverfassungsgericht unter Hinweis auf die Pressefreiheit aufgehoben und zur erneuten Prüfung an den BGH zurück verwiesen worden.

Am 12. Dezember beschließt das Europäische Parlament über den offenen MHPStandard: Er wird nicht vorgeschrieben, es soll jedoch die „Interoperabilität“ von interaktiven Digital-Fernsehdiensten und entsprechenden Geräten gefördert werden.

\section{Medienunternehmen}

Die Kirch-Gruppe verkauft mit Wirkung vom 1. Januar den im Austausch gegen eine Beteiligung an KirchMedia von Rupert Murdoch erworbenen Fernsehveranstalter TM3 an die Euvia Media AG \& Co. KG, eine Tochtergesellschaft der H.O.T. Networks. Ziel ist ein 24-stündiges Live-Programm mit Zuschauerbeteiligung per Call-in, bei dem Erlöse nicht nur aus Werbung, sondern auch aus Transaktionen wie z. B. der gebührenpflichtigen Teilnahme an Gewinnspielen oder Votings erzielt werden sollen. Im Mai erwirbt die ProSiebenSat.1 Media AG 48,4 Prozent der Euvia Media AG.

Am 11. Januar gibt die RTL Group bekannt, dass sie ihren verbliebenen 5-ProzentAnteil an Premiere zu einem Preis von 124 Mio. Euro an KirchPayTV verkauft hat. Sie übt damit eine Option aus, die bereits im März 1999 zwischen Kirch und CLT-UFA, der Vorgängerin der RTL Group, vereinbart worden war.

Die Gebühreneinzugszentrale (GEZ) teilt am 31. Januar mit, dass zwischen Ende 1998 und Ende 2000 die Gebühreneinnahmen der öffentlich-rechtlichen Rundfunkanstalten 450 Mio. DM höher ausgefallen sind als erwartet. Die Steigerung wird auf ihre Werbekampagne und die vermehrten Anmeldungen von Empfangsgeräten zurückgeführt. Nach Schätzung der GEZ sind mehr als 95 Prozent der Haushalte in Deutschland bei ihr angemeldet. Davon sind 7,7 Prozent von der Gebührenpflicht befreit.

Am 5. Februar teilt die Bertelsmann AG mit, dass sie von der Investmentgesellschaft Group Bruxelles Lambert (GBL) den 30-Prozent-Anteil an der RTL Group (Umsatz 2000 ca. 8 Mrd. DM) erwirbt. Im Gegenzug erhält die GBL einen Anteil von 25,1 Prozent an dem fast viermal so großen Bertelsmann-Konzern (Umsatz 2000 ohne RTL Group ca. 30 Mrd. DM). Dieser Anteil darf nach einigen Jahren an die Börse gebracht oder an Einzelinteressenten verkauft werden, allerdings mit Vorkaufsrecht der Bertels-

5 Abgedruckt in epd medien 89/2001. 
mann AG. Damit wird die Bertelsmann-Stiftung 57,6 Prozent der Kapitalanteile an der Bertelsmann AG halten, 17,3 Prozent die Familie Mohn und 25,1 Prozent die GBL. Der Erwerb wird am 11. Mai von der Europäischen Kommission genehmigt.

Am 15. März übernimmt die Kirch-Gruppe gemeinsam mit EM.TV die Mehrheit an SLEC, der Betreibergesellschaft der Formel 1-Autorennen. Vor diesem Hintergrund vereinbaren am 18. Mai fünf europäische Autohersteller, die an den Rennen mitwirken, die Gründung einer alternativen Betreibergesellschaft für Autorennen. Sie wollen sicherstellen, dass solche Rennen nicht nur im Pay-TV, sondern weiterhin im frei empfangbaren Fernsehen gezeigt werden.

Am 1. Mai erklären die Musik-Konzerne BMG und EMI, die zu den fünf größten der Branche gehören, dass sie ihr Fusionsvorhaben aufgeben. Die EU-Kommission hatte eine ablehnende Haltung gezeigt. Für den Fall einer Genehmigung wurden deshalb einschneidende Auflagen der Kartellbehörde erwartet.

Am 11. Mai beschließt der Verwaltungsrat des SWR eine Umstrukturierung der Fernsehproduktion. Ein Großteil der szenischen Produktionen des SWR soll künftig nicht mehr als Eigenproduktion, sondern über die Maran Film GmbH, ein gemeinsames Unternehmen des SWR und der Bavaria Film GmbH, abgewickelt werden. Im Vorfeld hatte der Bundesverband deutscher Fernsehproduzenten auf eine Protokollnotiz zum Rundfunkgebührenstaatsvertrag hingewiesen, nach der ein wesentlicher Teil der Gebührenerhöhung für die Herstellung neuer Werke durch freie Film- und Fernsehproduzenten verwendet werden soll. Die Auslagerung dürfe nicht dazu dienen, dass der SWR auf dem Umweg über Tochterunternehmen Fördermittel erhalte, die für die freie Produktionswirtschaft vorgesehen sind.

Am 2. Juli stellt Kabel New Media Insolvenzantrag. Das Hamburger Unternehmen war im Jahre 2000 noch die größte Internet-Agentur Deutschlands mit rund 1.000 Mitarbeitern und 136,5 Mio. DM Umsatz.

Am 4. September gibt die Deutsche Telekom den vollständigen Verkauf der KabelTochter KeTeKS mit den noch verbliebenen sechs regionalen Kabelgesellschaften an Liberty bekannt. Insgesamt sind in diesen Regionen gut 10 Mio. Haushalte angeschlossen, weitere 5 Mio. sind anschließbar. Der Kaufpreis beträgt 5,5 Mrd. Euro, davon $3 \mathrm{Mrd}$. in bar, 2,5 Mrd. in Aktien und $1 \mathrm{Mrd}$. als zehnjährige Schuldverschreibung. Der Vertrag steht unter dem Vorbehalt der Genehmigung durch das Bundeskartellamt. Die DLM weist darauf hin, dass hier nicht nur kartellrechtliche, sondern auch medienrechtliche Fragen zu prüfen sind.

Am 7. September teilt die Kirch-Gruppe mit, dass KirchMedia und ProSiebenSat.1 $A G$ zu einem Unternehmen verschmolzen werden sollen. Der Zusammenschluss soll bis Juni 2002 vollzogen werden. Er steht noch unter dem Vorbehalt der Zustimmung der Aufsichtsgremien der beiden Unternehmen. Infolge der Fusionsgerüchte ist die Aktie der ProSiebenSat.1 AG am 6. September um 20 Prozent eingebrochen.

Unmittelbar nach den Terror-Anschlägen auf New York und Washington am 11. September ergänzt der Axel-Springer-Verlag seine Unternehmensgrundsätze um die „Unterstützung des transatlantischen Bündnisses und die Solidarität in der freiheitlichen Wertegemeinschaft mit den Vereinigten Staaten von Amerika“.

Auf den Münchener Medientagen erklärt Miranda Curtis, die Präsidentin von Liberty Media International, am 19. Oktober, dass Liberty nicht die Absicht habe, Set-TopBoxen nach dem neuen MHP-Standard einzusetzen. Gleiches verlautet auch von Vertretern der Kabelnetzbetreiber iesy (vormals eKabel Hessen) und ish (vormals Kabel NRW). Liberty will stattdessen den Kabelkunden eine Dekoder-Box kostenlos zur Verfügung stellen. Auch der von der Politik gewünschte rasche Ausbau der Kapazitäten auf 
$862 \mathrm{MHz}$, wie er von Callahan in Bayern und Baden-Württemberg betrieben wird, sei nicht finanzierbar.

Am 13. November gibt die Deutsche Bank bekannt, dass sie einen Teil ihres Kabelunternehmens Tele Columbus an Liberty verkauft. Insgesamt hätte Liberty damit den Direktzugang zu 4,5 Mio. der gegenwärtig 10,1 Mio. Kabelhaushalte in diesen Ländern. Im Gegenzug wird eine Beteiligung der Bank an der Liberty Kabel Deutschland GmbH beschlossen. Beide Vereinbarungen stehen unter dem Vorbehalt der Genehmigung durch das Kartellamt. Noch im November meldet Liberty Media beim Kartellamt auch die Absicht einer Beteiligung bei BSkyB Germany an, das 22 Prozent der Anteile an Premiere hält.

\section{Medienangebote}

\subsection{Presse}

Am 20. Januar erscheint in Freiburg eine neue Tageszeitung des Verlegers Michael Zäh mit dem Titel zus. Wegen unzureichender Abonnentenzahlen und Anzeigenerlöse wird das Blatt nach drei Monaten wieder eingestellt.

Mitte des Jahres wird die Zeitschrift $e$-Business, ein Ableger der Wirtschaftswoche, wegen des zurückgehenden Anzeigengeschäfts eingestellt. Die Zeitschrift Net-Business der Verlagsgruppe Milchstraße, im Frühjahr zunächst von wöchentlichem auf vierzehntägliches Erscheinen umgestellt, wird im August eingestellt. Damit hat die Krise der IT-Branche auch einschlägig spezialisierte Zeitschriften erfasst.

Am 11. Juli wird die Kölner Gratiszeitung 20 minuten Köln der 20 min Holding AG des norwegischen Medienkonzerns Schibsted AS eingestellt, weil sich die Werbeeinnahmen nicht erwartungsgemäß entwickeln. Wenige Tage später werden die als Abwehrmaßnahme gegen den Marktzutritt konzipierten Gratiszeitungen Extra Köln des Axel-Springer-Verlages und Kölner Morgen der Zeitungsgruppe DuMont Schauberg ebenfalls eingestellt. Am 11. Mai hatte das Oberlandesgericht Köln eine Klage gegen die Gratiszeitung abgewiesen. Eine relevante Störung des Zeitungswettbewerbs könne derzeit nicht angenommen werden.

Am 12. September bringt der Axel-Springer-Verlag eine neue Kaufzeitung Extra für eine Zielgruppe im Alter von 14 bis 30 Jahren auf den Markt. Das Blatt wird im RheinNeckar-Raum getestet und am 10. Oktober wieder eingestellt, weil der nötige Absatz nicht erreicht wurde.

Am 30. September beginnt für die Frankfurter Allgemeine Sonntagszeitung der bundesweite Vertrieb mit einer Auflage von 600.000 Exemplaren. Der Verkauf erfolgt über Kioske, Tankstellen und Bäckereien sowie über die eigenen Vertriebsagenturen. 200.000 der 280.000 Abonnenten der FAZ erhalten das Blatt für vier Wochen kostenlos zur Ansicht.

\subsection{Rundfunk}

Zum Jahresanfang geht ARD Digital in den Regelbetrieb über. Das per Kabel und Satellit verbreitete Bouquet umfasst 18 Fernseh- und 22 Hörfunkprogramme. Drei der Fernsehprogramme werden ausschließlich digital übertragen: Eins-Extra bringt stündliche Nachrichten und weitere Informationsangebote, Eins $M u X x$ sendet das erste Programm zeitversetzt und EinsFestival zeigt Filme und Serien.

Mit dem 1. Januar übernimmt der Hessische Rundfunk zusätzlich zu den bisherigen 
vier Hörfunkprogrammen vier weitere Programme in den Regelbetrieb: Das Jugendradio br XXL, das Wirtschaftsradio br skyline, die Klassikwelle sowie das Nachrichtenradio hr chronos. Sie waren zunächst als digitale Zusatzprogramme gestartet. Inzwischen werden sie terrestrisch per Mittelwelle oder UKW ausgestrahlt oder per live streaming im Internet verbreitet. Die terrestrische digitale Verbreitung nach dem DAB-Standard wird eingestellt.

Am 16. Januar erteilt die Medienanstalt Berlin-Brandenburg dem von Spiegel-TV und dctp geplanten Metropolenfernsehen eine Zulassung für die Kabelverbreitung. Zugleich wird die Kabelbelegung so geändert, dass das Programm von 15 bis 7 Uhr auf einem analogen Kanal verbreitet werden kann. Unter dem Namen XXP geht es am 7. Mai auf Sendung. Besonderes Kennzeichen ist eine vertikal gegliederte Programmstruktur mit Themenschwerpunkten, die von Woche zu Woche wechseln.

Am 1. März startet RTL Shop, nach H.O.T. und QVC der dritte deutsche Teleshopping-Kanal. Beteiligt sind RTL Television (55\%), deren Tochter RTL Newmedia (25\%) und die französische Sendergruppe M6 (20\%). Das Programm wird über Astra 1 A verbreitet und erreicht damit etwa ein Drittel der Fernsehhaushalte. Zusätzlich werden morgens Programmfenster bei RTL, RTL2, Vox und Onyx genutzt. Auch H.O.T. startet im März Teleshopping-Fenster im Nachtprogramm und im Morgenprogramm bei Sat.1 und Kabel 1.

Am 3. Mai einigt sich eine Arbeitsgruppe von Deutscher Welle, ARD und ZDF auf ein Konzept für einen gemeinsamen deutschsprachigen Auslandskanal, der neben das dreisprachige Auslandsfernsehen der Deutschen Welle treten soll. Das Programm mit dem Namen German TV soll zunächst in den USA verbreitet werden und in einer Programmschleife von acht Stunden pro Tag Sendungen von ARD, ZDF und Deutscher Welle enthalten. Die Kosten sollen in den USA durch Pay-TV erbracht und im Übrigen vom Bund getragen werden. In der weiteren Diskussion wird aus dem Bundesrechnungshof die Befürchtung laut, dass die vorgesehene Anschubfinanzierung von 60 Mio. DM und ein jährlicher Bundeszuschuss von 5,5 Mio. DM nicht ausreichen werden. Der Haushaltsausschuss des Bundestages bewilligt am 15. November eine Anschubfinanzierung von 40 Mio. DM. Der Intendant der Deutschen Welle erklärt daraufhin, ohne eine Zusage über die notwendigen $60 \mathrm{Mio}$. DM könne er das Projekt nicht beginnen.

Mit dem Beginn der neuen Bundesligasaison am 28. Juli verlegt Sat.1 seine FußballSendung ran samstags von 18.30 Uhr auf 20.15 Uhr, offenbar aus Rücksicht auf die Verwertung im Pay-TV. Dies führt zum Streit zwischen der ARD und der Kirch-Gruppe über die Kurzberichte, die in der Tagesschau ab 20.00 Uhr gezeigt werden dürfen. Wegen erheblicher Einbußen bei den Marktanteilen von Sat.1 wird die Sendung jedoch ab dem 8. September wieder auf 19 Uhr vorgezogen. Damit wird auch der Konflikt über die Kurzberichte gegenstandslos.

Am 1. September startet ein privater Kanal mit dem Namen ChannelD als deutschsprachiges Pay-TV für Nordamerika, die Karibik und weite Teile Südamerikas. Das Unternehmen hat seinen Sitz in Bremerhaven und eine Zulassung der Bremischen Landesmedienanstalt. Sie ist zwar nicht erforderlich, kann aber für die Kabelverbreitung in den USA nützlich sein. Die Programmrechte werden von öffentlich-rechtlichen und privaten Veranstaltern erworben.

Die Berichterstattung über die Terror-Anschläge in New York und Washington am 11. September ab 14.48 Uhr MESZ dominiert ab dem späten Nachmittag die deutschen Fernsehprogramme. Dauersendungen zum aktuellen Geschehen werden auf die verschiedenen Programme der Senderketten durchgeschaltet. Vor diesem Hintergrund wird die Ausstrahlung von Fernsehwerbung drastisch verringert, die Bruttowerbeum- 
sätze erreichen an diesem Tag mit 5,4 Mio. Euro nur ein Viertel der Werte in den Tagen zuvor. Auch im weiteren Verlauf gibt es Änderungen in der Programmgestaltung. Comedy-Sendungen, Shows sowie Action- und Katastrophenfilme werden aus dem Programm gestrichen.

Am 1. Oktober nimmt das landesweite private Fernsehprogramm tv.nrw in Nordrhein-Westfalen seinen Sendebetrieb im Kabel auf. Gesellschafter der Betreibergesellschaft sind die drei größten Zeitungsverlage Nordrhein-Westfalens: die Zeitungsgruppe WAZ aus Essen, die Verlagsgruppe Rheinische Post aus Düsseldorf und das Verlagshaus DuMont Schauberg aus Köln.

Am 1. November starten Radio Bremen und NDR das gemeinsame Hörfunkprogramm Nordwest-Radio. Es tritt an die Stelle der bisherigen Kulturwelle von Radio Bremen, die wegen der zurückgehenden Einnahmen aus dem ARD-Finanzausgleich so nicht fortgesetzt werden konnte. Das Projekt ist zunächst bis 2005 befristet.

Am 26. November legt die Arbeitsgemeinschaft der Landesmedienanstalten (ALM) ihren 3. Programmbericht zur Lage und Entwicklung des Fernsehens vor. Danach ist die Programmentwicklung auf vielen Kanälen durch eine geringere Programmleistung geprägt, stattdessen gibt es mehr Werbung, mehr Teleshopping und mehr kurzfristige Wiederholungen. Die Unterhaltung dominiert, aber neben die Filme und Serien treten zunehmend Quizsendungen, Shows und Spiele sowie unterhaltende Publizistik etwa in Form von Talkshows. Die politische Informationsleistung des Fernsehens ist rückläufig. In keinem der untersuchten privaten Programme hat der Anteil der politischen Publizistik im weit gefassten Sinne mehr als vier Prozent der Sendezeit betragen. Bei ARD und ZDF ist der Anteil der politischen Publizistik auf 18 Prozent zurückgegangen.

\subsection{Online-Medien}

Am 29. Januar teilt die Kirch-Gruppe mit, dass der Start der von Kirch New Media entwickelten Entertainment-Plattform maxdome auf unbestimmte Zeit verschoben und die Gesellschaft mit ProSieben Digital Media zusammengelegt werden soll. Das Unternehmen soll sich auf den Ausbau des vorhandenen Online-Marken-Netzwerkes beschränken. Fast zeitgleich wird bekannt gegeben, dass die Bertelsmann Broadband Group in RTL New Media integriert wird. Wegen der Verzögerungen im Verkauf und im Ausbau des Kabelnetzes werden vorerst zu geringe Chancen für Inhalte-Anbieter gesehen.

Am 4. April startet www.bild.t-online.de, ein Joint Venture des Axel-Springer-Verlages mit der T-Online AG, die 37 Prozent der Anteile hält. Beide sind in ihrem Bereich Marktführer in Deutschland, Bild mit 11 Mio. Lesern, T-Online mit 14 Mio. Nutzern. Ein Kooperationsprojekt von T-Online und ZDF unter der Adresse www.heute.t-online.de startet am 25. August. Der Bundesverband Deutscher Zeitungsverleger kritisiert dieses Projekt als rechtswidrig.

Am 9. August teilen die Verlagsgruppen Burda und Milchstraße mit, dass sie ihre Internet-Portale zusammenlegen und die Focus Digital AG und die Tomorrow Internet AG zur Tomorrow Focus AG fusionieren. Sie reagieren damit auf die zurückgehenden Erwartungen im Online-Sektor.

\section{Werbung}

Für das Gesamtjahr 2001 gehen die Bruttowerbeeinnabmen der Medien um 6,3 Prozent auf $17 \mathrm{Mrd}$. Euro zurück, ermittelt die AC Nielsen Werbeforschung S+P. Betroffen sind alle klassischen Medien, am stärksten Fachzeitschriften (-15,9\%), Zeitungen (-10,5\%) 
und Hörfunk (-10,5\%). Gegenüber 1999 verbleibt ein Zuwachs von 4,9 Prozent. Der langfristige Trend zugunsten der elektronischen Medien setzt sich fort: Sie erreichen einen Marktanteil von 49,9 Prozent, der Anteil der Presse verringert sich auf 47,3 Prozent, die Plakatwerbung erzielt mit 2,7 Prozent ein nahezu konstantes Ergebnis.

Zum 1. Juli führt der Heinrich Bauer Verlag ein neues Anzeigen-Reservierungssystem ein, mit dem es möglich ist, bis zu einem halben Jahr im Voraus Anzeigenplätze in Zeitschriften online zu reservieren. Unter www.adplacement.de sind dazu auch Informationen über die geplante redaktionelle Struktur und die Branchenzuordnung der bereits vorliegenden Reservierungen einsehbar.

\section{Mediennutzung}

Die Deutschen verbringen im Durchschnitt knapp achteinhalb Stunden pro Tag mit der Mediennutzung. Das zeigen die im April publizierten Ergebnisse der Langzeitstudie „Massenkommunikation“, die seit 1964 in mehrjährigen Abständen von ARD und ZDF durchgeführt wird. Mit 206 Minuten täglich entfällt die meiste Zeit auf den Hörfunk; mit 185 Minuten folgt das Fernsehen. Die Zeitungslektüre beschäftigt die Menschen eine halbe Stunde pro Tag, Bücher 18 Minuten, Zeitschriften 10 Minuten, das Internet im Durchschnitt 13 Minuten. Die Musik-Speichermedien CD, MC und LP werden zusammen 36 Minuten pro Tag genutzt, der Video-Konsum umfasst 4 Minuten täglich.

Im September legen ARD und ZDF neue Ergebnisse aus einer Untersuchung der Internet-Nutzung vor. Danach sind in Deutschland 24,8 Mio. Erwachsene online. Das Internet wird von 38,8 Prozent der Deutsch sprechenden Bevölkerung ab 14 Jahren genutzt. Deutliche Veränderungen zeigen sich hinsichtlich des Nutzungsortes: 1997 war die Internet-Nutzung noch überwiegend an den Arbeitsplatz oder den Ausbildungsort gebunden. Inzwischen haben 78 Prozent der User einen Internet-Zugang in ihrer häuslichen Umgebung.

Zum 31. Oktober scheidet die Motor-Presse Stuttgart, der größte Verlag für Special Interest-Zeitschriften in Europa, aus der Informationsgemeinschaft zur Feststellung der Verbreitung von Werbeträgern (IVW) aus und kommt einem Ausschlussverfahren wegen falscher Meldungen zuvor. Als Folge werden auch bei der Media-Analyse keine Daten über die Zeitschriften dieses Verlages mehr ausgewiesen. Die Zeitschrift modern living, deren Auflage um mehr als 80 Prozent überhöht gemeldet wurde, wird eingestellt.

\section{Quellen}

epd medien, Funkkorrespondenz, medien aktuell, Media Perspektiven, Presseinformationen von Medienunternehmen, Verbänden und Landesmedienanstalten, eigene Recherchen 